\title{
Perfil Sociodemográfico e Clínico de Pacientes com Câncer Cadastrados no Programa de Visita Domiciliar de um Hospital da Rede Pública
}

doi: https://doi.org/10.32635/2176-9745.RBC.2021v67n2.864

\author{
Sociodemographic and Clinical Profile of Cancer Patients Registered in the Home Visiting Program of a Public Hospital \\ Perfil Sociodemográfico y Clínico de los Pacientes Oncológicos Registrados en el Programa de Visitas Domiciliarias de \\ un Hospital Público
}

Adrielly Elane Sousa Maia'; Flávia Adrianne de Castro Grello²; Katiane da Costa Cunha ${ }^{3}$

RESUMO

Introduçáo: O perfil sociodemográfico constitui fator de vulnerabilidade para o desenvolvimento de neoplasias em geral, podendo comprometer as açóes de prevenção, dificultar o diagnóstico precoce e/ou acesso à terapêutica adequada, ocasionando reflexos negativos no prognóstico e na qualidade de vida dos pacientes. O cuidado domiciliar tem seu planejamento iniciado no ambiente hospitalar, cuja família/cuidador recebe orientaçóes acerca do enfrentamento da doença e, sobretudo, da prevenção de complicaçóes relacionadas com o estado patológico. Objetivo: Caracterizar o perfil sociodemográfico e clínico de pacientes com câncer cadastrados do programa da visita domiciliar de um hospital da rede pública. Método: Estudo transversal, quantitativo, retrospectivo e descritivo, cujos dados foram extraídos da análise de 274 prontuários de pacientes oncológicos cadastrados no serviço de visita domiciliar do hospital, no período de 2010 a 2017. Resultados: A maioria dos pacientes era do sexo feminino na faixa etária de 60 a 80 anos, com média de estudo de quatro a sete anos e residentes na cidade de Belém. O câncer geniturinário foi o mais frequente e classificado no estágio IV. Por fim, a maior parte dos pacientes apresentava dor, fazendo o uso de analgésicos de ampla potência. Conclusáo: Torna-se importante conhecer o perfil clínico e sociodemográfico dos pacientes, para melhor planejamento e intervenção junto à equipe multidisciplinar, uma vez que a população no Estado do Pará tem um cenário de alta prevalência dos tipos de câncer que podem ser diagnosticados precocemente.

Palavras-chave: Neoplasias/epidemiologia; Serviços de Assistência Domiciliar; Cuidados Paliativos; Estudos Transversais.

\section{ABSTRACT}

Introduction: The sociodemographic profile is a vulnerability factor for the development of neoplasms in general, which can compromise preventive actions, hinder early diagnosis and/or access to an appropriate therapeutic, causing negative effects on the prognosis and quality of life of patients. Home care planning initiates in the hospital environment, when the family/ caregiver receives guidance about coping with the disease and, above all, preventing complications related to the pathological condition. Objective: To characterize the sociodemographic and clinical profile of cancer patients registered in the home visit program of a public hospital. Method: Cross-sectional, quantitative, retrospective, and descriptive study whose data were extracted from the analysis of 274 charts of cancer patients registered in the hospital's home visit service from 2010 to 2017. Results: Most of the patients were females in the age range from 60 to 80 years old, mean of 4 to7 years of formal education and living in the city of Belém. Stage IV genitourinary cancer was the most frequent. Finally, most patients had pain and were using wide-range potency analgesics. Conclusion: It is important to know the clinical and sociodemographic profile of the patients, in order to better planning and intervention together with the multidisciplinary team, since the population in the State of Pará has a scenario of high prevalence of types of cancer that can be diagnosed early.

Key words: Neoplasms/epidemiology; Home Care Services; Palliative Care; Cross-Sectional Studies.

\section{RESUMEN}

Introducción: El perfil sociodemográfico es un factor de vulnerabilidad para el desarrollo de neoplasias en general, que puede comprometer acciones preventivas, dificultar el diagnóstico precoz y/o el acceso a la terapia adecuada, provocando efectos negativos sobre el pronóstico y la calidad de vida de los pacientes. La atención domiciliaria tiene su planificación iniciada en el ámbito hospitalario, en el que el familiar/cuidador recibe orientación sobre el afrontamiento de la enfermedad y, sobre todo, la prevención de complicaciones relacionadas con el estado patológico. Objetivo: Caracterizar el perfil sociodemográfico y clínico de los pacientes oncológicos registrados en el programa de visitas domiciliarias de un hospital público. Método: Estudio transversal, cuantitativo, retrospectivo y descriptivo, cuyos datos se extrajeron del análisis de 274 historias clínicas de pacientes oncológicos registrados en el servicio de visita domiciliaria del hospital de 2010 a 2017. Resultado: La mayoría de los pacientes eran del sexo masculino mujer de 60 a 80 ańos, con un estudio promedio de cuatro a siete años y residente en la ciudad de Belém, siendo el cáncer genitourinario el más frecuente clasificado en estadio IV. Finalmente, la mayoría de los pacientes experimentaron dolor al usar analgésicos de gran potencia. Conclusión: Es importante conocer el perfil clínico y sociodemográfico de los pacientes, para poder planificación e intervención mejor con el equipo multidisciplinario, ya que la población del Estado de Pará tiene un escenario de alta prevalencia de tipos de cáncer que pueden ser diagnosticado temprano.

Palabras clave: Neoplasias/epidemiologia; Servicios de Atención de Salud a Domicilio; Cuidados Paliativos; Estudios Transversales.

\footnotetext{
1,2Hospital Ophir Loyola (HOL). Programa de Residência Multiprofissional em Oncologia - Cuidados Paliativos. Belém (PA), Brasil. E-mails: adriellymaiaa@gmail.com; flaviaadrienne@hotmail.com. Orcid iD: http://orcid.org/0000-0002-6940-2417; Orcid iD: http://orcid.org/0000-0003-3031-6682

${ }^{3}$ Universidade Federal do Pará (UFPA). Belém (PA), Brasil. E-mail: katianefisio@yahoo.com.br. Orcid iD: https://orcid.org/0000-0001-5361-5090

Endereço para correspondência: Adrielly Elane Sousa Maia. Ed. Torres Devant. Travessa Pirajá, 520, apto. 1202 - A - Leste - Pedreira. Belém (PA), Brasil. CEP 66083-513. E-mail: adriellymaiaa@gmail.com
} 


\section{INTRODUÇÃO}

O câncer é definido como o crescimento desordenado de células que invadem tecidos e orgáos, classificadas como malignas, além de ocasionar a disseminação pelas diferentes regióes do corpo, longe do local de origem, denominada de metástase ${ }^{1}$.

Atualmente, o câncer é considerado um problema de saúde pública e vem ganhando cada vez mais atenção em razáo do envelhecimento populacional, pois a idade está relacionada a diversas alteraçôes fisiológicas e a outros fatores próprios do mundo moderno, como o estilo de vida inadequado. $\mathrm{O}$ melhor prognóstico para o paciente geralmente está relacionado com o estadiamento, precocidade do diagnóstico, tipo de neoplasia e tratamento adequado $^{1-3}$.

Porém, por vezes, o diagnóstico é feito tardiamente, com repercussões negativas no sucesso da terapêutica, sendo indispensável a inserção de cuidados paliativos como terapêutica complementar ou exclusiva, que objetiva amenizar sinais e sintomas, promovendo o bem-estar do paciente e cuidador ou familiar ${ }^{4}$.

O câncer é uma doença crônica e um marcador de saúde no Brasil; assim, é utilizado como indicador do grau de desenvolvimento regional em razão das reflexóes sobre as neoplasias e suas questóes demográficas acerca das condiçôes de vida e desenvolvimento da população ${ }^{5}$.

A literatura aponta que o perfil sociodemográfico pode aumentar a vulnerabilidade para o desenvolvimento do câncer no acesso às açôes e procedimentos de prevenção, no tempo para definição de diagnóstico, na escolha da modalidade terapêutica e, portanto, no prognóstico e sobrevida dos pacientes ${ }^{6,7}$.

As políticas de controle de câncer no país têm como base o perfil de morbidade e mortalidade dos diversos Estados e municípios do país, com ampla variaçáo de Região para Região. Com base nas estimativas de casos incidentes de câncer, podem-se oferecer informaçóes epidemiológicas que são fundamentais para o planejamento de açóes de promoção à saúde, detecção precoce e de atenção oncológica em todos os níveis 5 .

Sendo uma ferramenta para o atendimento ao paciente, principalmente aqueles com doenças avançadas e em fase terminal, o cuidado paliativo é a ação multiprofissional e interdisciplinar que proporciona atenção integral e ativa ao indivíduo. Em relação ao suporte domiciliar, há uma rede assistencial que visa à desospitalização, à prevenção de riscos e à humanização da assistência, e oferece, ao paciente, maior controle dos sintomas mais estressores e uma qualidade em seu fim de vida, em um ambiente onde, dentro do possível, suas preferências sejam priorizadas ${ }^{8,9}$.
A internação domiciliar é um recurso do cuidado paliativo, sendo solicitada frequentemente por parte dos pacientes e/ou familiares, ocorrendo principalmente em indivíduos em fase terminal. Importante ressaltar que essa ação não é definitiva e requer diversas reavaliações, por causa do estado lábil ${ }^{9}$.

Esse cuidado tem seu planejamento inicial no ambiente hospitalar, como proposta da equipe multiprofissional, onde ocorre a deliberação sobre as condiçóes de receber tal acompanhamento em anuência com a família/ cuidador, sendo esta informada sobre as necessidades e responsabilidade frente ao cuidado, assim como sobre as possíveis limitaçôes e a forma de implementação. Em geral, ocorre de forma gradual, não linear, sendo acompanhada e reavaliada de acordo com seu quadro clínico, com periodicidade variada, a cada mês, em quinzenas, semanal ou até mesmo diária ${ }^{10}$.

Para que haja uma boa assistência domiciliar, são necessários critérios de inclusão de pacientes de forma clara e efetiva, para maximizar o tempo e evitar a negligência de situaçóes que exigem cuidado no domićlí. Visando a organizar essas açóes, a Portaria n. ${ }^{\circ} 825$, de 25 de abril de $2016^{11}$, em seu capítulo II, traz as indicações de atenção domiciliar para pacientes que possuem estabilidade clínica e precisam de cuidados à saúde, seja de modo temporário, definitivo, ou para os que estão de certa forma mais vulneráveis, sendo a assistência mais apropriada para tratamento, reabilitação, prevenção de agravos e cuidados paliativos.

Outra ferramenta de auxílio nesse cuidado é o registro em saúde, por meio do prontuário, que também contribui para a decisão clínica e gerencial, acompanhamento e continuidade do processo de assistência fornecida, além de ser o elo de comunicação entre os diversos profissionais, fonte da história pregressa, clínica e atual dos pacientes, sendo sua implementação e conteúdo critérios de avaliação da qualidade da prestação de serviço da saúde ${ }^{12}$.

Assim, este estudo tem como objetivo analisar o perfil sociodemográfico e clínico de pacientes portadores de câncer, atendidos no programa de visita domiciliar do Hospital Ophir Loyola, no período de 2010 a 2017, gerando evidências que contribuem com o aprofundamento do conhecimento acerca dessa temática, uma vez que a aproximaçáo com a realidade vivenciada pelo paciente, que está em cuidado domiciliar, poderá contribuir no processo de atenção à saúde para a equipe multiprofissional.

\section{MÉTODO}

Estudo de caráter transversal, quantitativo, retrospectivo e descritivo da análise dos prontuários de pacientes 
oncológicos atendidos no programa de visita domiciliar do Hospital Ophir Loyola, no período de 2010 a 2017. O período da pesquisa compreende os meses de dezembro de 2018 a abril de 2019.

Este trabalho foi realizado de acordo com os aspectos éticos preconizados na Declaração de Helsinque e Código de Nuremberg, e pela norma de pesquisa que envolve seres humanos do Conselho Nacional de Saúde (Resolução CNS 466/12) ${ }^{13}$. Iniciou após a autorizaçáo do Comitê de Ética em Pesquisa com Seres Humanos do Hospital Ophir Loyola, que possui um programa de visitas domiciliares para o acompanhamento de pacientes oncológicos, onde são realizados atendimentos e visitas regulares em domicílio em diversos bairros da cidade de Belém, com número de parecer 3.009.002, para acesso livre aos prontuários.

Foram inclusos na pesquisa apenas prontuários de pacientes oncológicos em cuidados paliativos atendidos no programa de visita domiciliar desse mesmo hospital nos últimos oito anos, sendo excluídos da pesquisa prontuários incompletos; ou seja, com informaçôes insuficientes para a composição do banco de dados.

A coleta de dados foi realizada por meio da análise das informaçóes contidas nos prontuários dos pacientes, mediante preenchimento de uma ficha de coleta de dados de autoria das pesquisadoras. Os dados coletados incluem: o valor da renda do núcleo familiar do paciente, número de pessoas na casa, cuidador principal, nível de escolaridade, sobrevida na visita domiciliar e a composiçáo da equipe multidisciplinar.

Foram analisados também o diagnóstico clínico, estadiamento da doença, presença de dor, tratamentos anteriores (radioterapia, quimioterapia, hormonioterapia e cirurgia), medicamentos utilizados e a presença de comorbidades, como metástases, hipertensão arterial sistêmica, diabetes mellitus, insuficiência renal aguda.

O levantamento bibliográfico foi realizado nas seguintes bases de dados: Biblioteca Virtual em Saúde (BVS), Scientific Electronic Library Online (SciELO) e Medical Literature Analysis and Retrievel System Online (MEDLINE) via PubMed. Os termos utilizados para a busca foram principalmente: câncer, análise de prontuários, visita domiciliar, cuidados paliativos e perfil sociodemográfico.

O software Excel 2010 foi adotado para entrada dos dados, bem como para a confecçáo das tabelas. A análise estatística foi realizada por meio dos softwares BioEstat 5.0 e EpiInfo 3.5.1. As variáveis categóricas foram apresentadas como frequências e as numéricas por meio de medidas de tendência central e dispersão. Utilizaram-se os testes qui-quadrado, qui-quadrado (contingência) e G (aderência) para avaliar a significância dos dados.
Todos os resultados foram considerados estatisticamente significativos no nível de significância de 5\% ( $\leq 00,05)$.

\section{RESULTADOS}

Dos 440 prontuários coletados no Hospital Ophir Loyola, dos pacientes que compóem o atendimento domiciliar, foram excluídos 166 por possuírem dados incompletos. Assim, a amostra final do estudo foi composta de 274 prontuários de pacientes cadastrados.

Dos 274 prontuários analisados, a maioria dos pacientes era do sexo feminino $\left(59,9 \%, \mathrm{p}=0,001^{*}\right)$, na faixa etária dos 60 a 80 anos $\left(50,0 \%, p<0,0001^{*}\right)$, casado $(45,3 \%$, $\left.\mathrm{p}<0,0001^{*}\right)$, com média de estudo de quatro a sete anos $\left(37,6 \%, \mathrm{p}<0,0001^{*}\right)$, tendo o filho o cuidador principal $\left(53,6 \%, \mathrm{p}<0,0001^{*}\right)$, especialmente do sexo feminino $\left(86,5 \%, \mathrm{p}<0,0001^{*}\right)$, conforme mostra a Tabela 1 .

Com relação à naturalidade, a maior parte era do Pará $\left(95,6 \%, \mathrm{p}<0,0001^{*}\right)$, pertencente à cidade de Belém $\left(93,4 \%, p<0,0001^{*}\right)$, com renda familiar de um a três salários-mínimos $\left(61,3 \%, \mathrm{p}<0,0001^{*}\right)$, onde de quatro a sete pessoas moram na mesma casa $\left(41,2 \%, \mathrm{p}<0,0001^{*}\right)$, conforme mostra a Tabela 2.

Foram evidenciados variados tipos de câncer entre os 274 prontuários analisados, os cânceres identificados foram classificados segundo o sistema comprometido. A maioria apresentou câncer no sistema geniturinário $\left(44,2 \%, \mathrm{p}<0,0001^{*}\right)$, maior quantitativo de admissão no ano de $2012\left(23,6 \%, \mathrm{p}<0,0001^{*}\right)$, com a última visita também em $2012\left(27,4 \%, \mathrm{p}<0,0001^{*}\right)$, estadiamento do câncer IV $\left(59,9 \%, \mathrm{p}<0,0001^{*}\right)$, e a maior parte apresentava dor $\left(76,3 \%, \mathrm{p}<0,0001^{*}\right)$ (Tabela 3$)$.

Entre as comorbidades analisadas, a maioria dos pacientes não as apresentaram $\left(56,9 \%, \mathrm{p}<0,0001^{*}\right)$. Porém, entre aqueles que apresentaram comorbidades, 22,3\% tinham hipertensão arterial sistêmica, 9,1\% diabetes mellitus e $4 \%$ insuficiência renal aguda. Dos indivíduos que tinham metástase, grande parte encontrava-se no sistema ósseo $(30,3 \%)$ e realizou radioterapia $(46,0 \%)$. Foi possível observar que, entre os profissionais pertencentes à única equipe multidisciplinar, o assistente social era quem estava mais presente $(96,0 \%)$, se comparado aos profissionais da reabilitação como o fisioterapeuta e o terapeuta ocupacional, que participavam das visitas apenas uma vez por mês (Tabela 4).

No que diz respeito ao desfecho clínico, 62,0\% vieram a óbito $\left(\mathrm{p}<0,0001^{*}\right)$ tanto em ambiente domiciliar quanto hospitalar. A maioria $(26,6 \%)$ teve o desfecho em 2012, com média de sobrevida para aqueles que vieram a óbito de $115,43 \pm 199,15$ dias.

Em relação à terapia medicamentosa, 54,0\% fizeram uso de analgésico de alta potência (Tabela 5). 
Tabela 1. Dados gerais dos pacientes com câncer, atendidos pelo programa de visita domiciliar do Hospital Ophir Loyola, Belém, Pará, 2010 a $2017(n=274)$

\begin{tabular}{|c|c|c|c|}
\hline VARIÁVEIS & $\mathbf{N}$ & $\%$ & p-valor \\
\hline \multicolumn{4}{|l|}{ Sexo } \\
\hline Masculino & 110 & $40,1 \%$ & \multirow{2}{*}{${ }^{a} 0,001 *$} \\
\hline Feminino & 164 & $59,9 \%$ & \\
\hline \multicolumn{4}{|l|}{ Idade } \\
\hline 18 a 38 anos & 16 & $5,8 \%$ & \multirow{5}{*}{${ }^{b}<0,0001^{*}$} \\
\hline 39 a 59 anos & 71 & $25,9 \%$ & \\
\hline 60 a 80 anos & 137 & $50,0 \%$ & \\
\hline 81 a 101 anos & 49 & $17,9 \%$ & \\
\hline 102 a 122 anos & 1 & $0,4 \%$ & \\
\hline \multicolumn{4}{|l|}{ Estado civil } \\
\hline Solteiro(a) & 46 & $16,8 \%$ & \multirow{5}{*}{${ }^{b}<0,0001^{*}$} \\
\hline Casado(a) & 124 & $45,3 \%$ & \\
\hline Divorciado(a) & 22 & $8,0 \%$ & \\
\hline Viúvo(a) & 58 & $21,2 \%$ & \\
\hline Não informado & 24 & $8,8 \%$ & \\
\hline \multicolumn{4}{|l|}{ Escolaridade } \\
\hline Sem instrução & 25 & $9,1 \%$ & \multirow{6}{*}{${ }^{b}<0,0001^{*}$} \\
\hline 4 a 7 anos & 103 & $37,6 \%$ & \\
\hline 8 a 10 anos & 55 & $20,1 \%$ & \\
\hline 11 a 14 anos & 35 & $12,8 \%$ & \\
\hline 15 ou mais anos & 12 & $4,4 \%$ & \\
\hline Não informado & 44 & $16,1 \%$ & \\
\hline \multicolumn{4}{|l|}{ Cuidador principal } \\
\hline Masculino & 34 & $12,4 \%$ & \multirow{3}{*}{${ }^{b}<0,0001^{*}$} \\
\hline Feminino & 237 & $86,5 \%$ & \\
\hline Não informado & 3 & $1,1 \%$ & \\
\hline \multicolumn{4}{|l|}{$\begin{array}{l}\text { Parentesco do } \\
\text { cuidador }\end{array}$} \\
\hline Cônjuge & 47 & $17,2 \%$ & \multirow{7}{*}{${ }^{b}<0,0001^{*}$} \\
\hline Mãe & 4 & $1,5 \%$ & \\
\hline Pai & 2 & $0,7 \%$ & \\
\hline Filho(a) & 147 & $53,6 \%$ & \\
\hline Família estendida & 67 & $24,5 \%$ & \\
\hline Amigo(a) & 2 & $0,7 \%$ & \\
\hline Cuidador profissional & 2 & $0,7 \%$ & \\
\hline
\end{tabular}

Legendas: $\mathrm{a}=$ Teste do qui-quadrado; $\mathrm{b}=$ Teste $\mathrm{G}$ (aderência). ${ }^{*}$ Resultado estatisticamente significante, $\mathrm{p} \leq 0,05$.

\section{DISCUSSÃO}

No presente estudo, foi verificado que o perfil de pacientes oncológicos, atendidos no programa de visita domiciliar do Hospital Ophir Loyola, era de mulheres,
Tabela 2. Dados sociodemográficos dos pacientes com câncer, atendidos pelo programa de visita domiciliar do Hospital Ophir Loyola, Belém, Pará, 2010 a 2017 ( $n=274$ )

\begin{tabular}{|c|c|c|c|}
\hline VARIÁVEIS & $\mathbf{N}$ & $\%$ & p-valor \\
\hline \multicolumn{4}{|l|}{ Naturalidade } \\
\hline Pará & 262 & $95,6 \%$ & \\
\hline Outro Estado & 11 & $4,0 \%$ & ${ }^{a}<0,0001^{*}$ \\
\hline Outro país & 1 & $0,4 \%$ & \\
\hline \multicolumn{4}{|l|}{ Cidade } \\
\hline Belém & 256 & $93,4 \%$ & ${ }^{b}<00001^{*}$ \\
\hline Ananindeua & 18 & $6,6 \%$ & \\
\hline \multicolumn{4}{|l|}{ Renda familiar } \\
\hline Nenhuma & 2 & $0,7 \%$ & \\
\hline < 1 salário-mínimo & 6 & $2,2 \%$ & \\
\hline $\begin{array}{l}1 \text { a } 3 \text { salários- } \\
\text {-mínimos }\end{array}$ & 168 & $61,3 \%$ & \\
\hline $\begin{array}{l}3 \text { a } 6 \text { salários- } \\
\text {-mínimos }\end{array}$ & 18 & $6,6 \%$ & \\
\hline $\begin{array}{l}6 \text { a } 9 \text { salários- } \\
\text {-mínimos }\end{array}$ & 1 & $0,4 \%$ & ${ }^{\mathrm{a}}<0,0001^{*}$ \\
\hline $\begin{array}{l}9 \text { a } 12 \text { salários- } \\
\text {-mínimos }\end{array}$ & 1 & $0,4 \%$ & \\
\hline $\begin{array}{l}\text { > } 12 \text { salários- } \\
\text {-mínimos }\end{array}$ & 1 & $0,4 \%$ & \\
\hline Não informado & 77 & $28,1 \%$ & \\
\hline \multicolumn{4}{|l|}{$\begin{array}{l}\text { Pessoas que moram } \\
\text { na mesma casa }\end{array}$} \\
\hline 1 a 3 pessoas & 96 & $35,0 \%$ & \\
\hline 4 a 7 pessoas & 113 & $41,2 \%$ & \\
\hline 8 a 10 pessoas & 11 & $4,0 \%$ & ${ }^{a}<0,0001^{*}$ \\
\hline$>10$ pessoas & 3 & $1,1 \%$ & \\
\hline Não informado & 51 & $18,6 \%$ & \\
\hline
\end{tabular}

Legendas: $\mathrm{a}=$ Teste $\mathrm{G}$ (aderência); $\mathrm{b}=$ Teste do qui-quadrado. ${ }^{*}$ Resultado estatisticamente significante, $\mathrm{p} \leq 0,05$.

resultado similar ao encontrado na literatura ${ }^{14}$. Existem altos índice de detecção de neoplasias típicas a esse gênero, como os cânceres do colo uterino e o de mama, que se destacam entre os tumores que afetam mulheres em todo o mundo, principalmente em regióes menos desenvolvidas. Já em países desenvolvidos, a incidência de câncer entre gêneros é similar por conta de investimentos em prevençãa ${ }^{15}$.

A faixa etária de maior predominância na amostra foi de 60 a 80 anos. Achado que coincide com um estudo no qual foram analisados 859 prontuários de pacientes, sendo a maior parte deles com idade média de 72 e a mediana de 78 anos $^{16}$. Outras duas pesquisas explicam tal fato pelo aumento da expectativa de vida; isto é, o envelhecimento populacional e a sua intima relação com as doenças 
Tabela 3. Caracterização clínica dos pacientes com câncer, atendidos pelo programa de visita domiciliar do Hospital Ophir Loyola, Belém, Pará, 2010 a 2017 (n=274)

\begin{tabular}{|c|c|c|c|}
\hline VARIÁVEIS & $\mathbf{N}$ & $\%$ & p-valor \\
\hline \multicolumn{4}{|l|}{ Diagnóstico clínico } \\
\hline $\begin{array}{l}\text { Câncer do sistema } \\
\text { digestório }\end{array}$ & 90 & $32,8 \%$ & \\
\hline $\begin{array}{l}\text { Câncer do sistema } \\
\text { cardiovascular }\end{array}$ & 12 & $4,4 \%$ & \\
\hline $\begin{array}{l}\text { Câncer do sistema } \\
\text { respiratório }\end{array}$ & 20 & $7,3 \%$ & \\
\hline $\begin{array}{l}\text { Câncer do sistema } \\
\text { geniturinário }\end{array}$ & 121 & $44,2 \%$ & \\
\hline Câncer do sistema nervoso & 8 & $2,9 \%$ & \\
\hline $\begin{array}{l}\text { Câncer do sistema } \\
\text { tegumentar }\end{array}$ & 4 & $1,5 \%$ & $<0,0001^{*}$ \\
\hline Câncer do sistema linfático & 3 & $1,1 \%$ & \\
\hline $\begin{array}{l}\text { Câncer do sistema } \\
\text { endócrino }\end{array}$ & 2 & $0,7 \%$ & \\
\hline Câncer do sistema ósseo & 7 & $2,6 \%$ & \\
\hline Câncer de partes moles & 1 & $0,4 \%$ & \\
\hline Tumor primário oculto & 4 & $1,5 \%$ & \\
\hline Não informado & 2 & $0,7 \%$ & \\
\hline \multicolumn{4}{|l|}{$\begin{array}{l}\text { Ano de admissão } \\
\text { hospitalar }\end{array}$} \\
\hline 2010 & 4 & $1,5 \%$ & \\
\hline 2011 & 38 & $13,9 \%$ & \\
\hline 2012 & 72 & $26,3 \%$ & \\
\hline 2013 & 37 & $13,5 \%$ & \\
\hline 2014 & 27 & $9,9 \%$ & $<0,0001^{*}$ \\
\hline 2015 & 25 & $9,1 \%$ & \\
\hline 2016 & 33 & $12,0 \%$ & \\
\hline 2017 & 35 & $12,8 \%$ & \\
\hline Não informado & 3 & $1,1 \%$ & \\
\hline \multicolumn{4}{|l|}{ Última visita } \\
\hline Em assistência & 7 & $2,6 \%$ & \\
\hline 2010 & 5 & $1,8 \%$ & \\
\hline 2011 & 19 & $6,9 \%$ & \\
\hline 2012 & 75 & $27,4 \%$ & $<0,0001^{*}$ \\
\hline 2013 & 43 & $15,7 \%$ & \\
\hline 2014 & 32 & $11,7 \%$ & \\
\hline 2015 & 25 & $9,1 \%$ & \\
\hline 2016 & 35 & $12,8 \%$ & \\
\hline 2017 & 33 & $12,0 \%$ & \\
\hline \multicolumn{4}{|l|}{ Estadiamento do câncer } \\
\hline III & 18 & $6,6 \%$ & \\
\hline IV & 164 & $59,9 \%$ & $<0,0001^{*}$ \\
\hline Não informado & 92 & $33,6 \%$ & \\
\hline \multicolumn{4}{|l|}{ Presença de dor } \\
\hline Sim & 209 & $76,3 \%$ & \\
\hline Não & 24 & $8,8 \%$ & $<0,0001^{*}$ \\
\hline Não informado & 41 & $15,0 \%$ & \\
\hline
\end{tabular}

Legendas: Teste $G$ (Aderência). * Resultado estatisticamente significante, $p \leq 0,05$. crônicas não transmissíveis (DCNT), além da exposição aos fatores de riscos ambientais, como o câncer, podendo gerar uma maior demanda por cuidados paliativos ${ }^{17,18}$.

Quanto à escolaridade, a média foi de quatro a sete anos de estudo e a renda familiar de um a três salários-mínimos, onde de quatro a sete pessoas moram na mesma casa. Dado também revelado no estudo de Lampert et al. ${ }^{19}$, no qual os resultados mostraram que a maioria possui até quatro anos de estudo, realidade que prejudica o entendimento das orientaçóes de saúde, dificuldade de acesso a programas preventivos, à assistência ao diagnóstico precoce e a tratamento adequado imediato. Quanto à renda familiar, as DCNT atingem de forma mais intensa grupos de maior vulnerabilidade, como os de baixa renda e escolaridade ${ }^{20}$.

Com relação à frequência do câncer na populaçáo estudada, a maioria está localizada no sistema geniturinário, o que pode ser em virtude dos altos índices dos cânceres de próstata e do colo uterino na Região Norte ${ }^{21}$. Dado similar ao encontrado no estudo realizado por Atty e Tomazelli ${ }^{22}$, em que, entre as principais neoplasias que demandaram cuidado paliativo no atendimento domiciliar em homens, o de próstata foi o mais recorrente e, em mulheres, o de mama. Os autores outrora citados ${ }^{22}$ ressaltam ainda que é preocupante a frequência de casos em cuidados paliativos por câncer do colo do útero, por ser uma neoplasia de fácil diagnóstico precoce e prevenção em toda atenção básica do país.

Para atender às necessidades das pessoas em cuidados paliativos, com metástases regionais ou a distância, além do tratamento sintomático, podem ser utilizados tratamentos com finalidade paliativa, como cirurgia, quimioterapia, radioterapia e hormonioterapia ${ }^{23}$. Dos tipos de tratamentos antineoplásicos realizados anteriormente pelos pacientes desta pesquisa, o que apresentou maior frequência foi a radioterapia, seguida da quimioterapia e cirurgia. Contudo, em um estudo ${ }^{24}$ com pacientes em estádio avançado, em um hospital de referência em Curitiba, a partir dos tratamentos antineoplásicos anteriores, foi constatado que 78 pacientes realizaram quimioterapia, 57 fizeram algum tipo de cirurgia e 42 pacientes realizaram radioterapia, 13 receberam outros tipos de tratamento, tais como hormonioterapia ou braquiterapia, e 30 não receberam tratamento curativo/paliativo.

Conforme observado nos resultados do presente estudo, os pacientes encontravam-se em estádio avançado da doença, sendo que, na literatura ${ }^{25}$, também é comum que a maioria dos pacientes, no momento da internação, esteja em estádios avançados da doença (mais de 90\% com metástases). Com isso, reforça-se a importância dos cuidados paliativos, juntamente com a necessidade de sua expansáo, para garantir a universalidade e a integralidade 
Tabela 4. Caracterização clínica dos pacientes com câncer, atendidos pelo programa de visita domiciliar do Hospital Ophir Loyola, Belém, Pará, 2010 a $2017(\mathrm{n}=274)$

\begin{tabular}{|c|c|c|c|c|c|}
\hline \multirow{2}{*}{ VARIÁVEIS } & \multicolumn{2}{|c|}{ Sim } & \multicolumn{2}{|c|}{ Não } & \multirow{2}{*}{ p-valor } \\
\hline & $\mathbf{N}$ & $\%$ & $\mathbf{N}$ & $\%$ & \\
\hline \multicolumn{6}{|l|}{ Comorbidades } \\
\hline Ausente & 156 & $56,9 \%$ & 118 & $43,1 \%$ & \\
\hline Hipertensão arterial sistêmica & 61 & $22,3 \%$ & 213 & $77,7 \%$ & \\
\hline Diabetes mellitus & 25 & $9,1 \%$ & 249 & $90,9 \%$ & $<0,0001^{*}$ \\
\hline Insuficiência renal aguda & 11 & $4,0 \%$ & 263 & $96,0 \%$ & \\
\hline Não informado & 38 & $13,9 \%$ & 236 & $86,1 \%$ & \\
\hline \multicolumn{6}{|l|}{ Metástase } \\
\hline Sistema digestório & 53 & $19,3 \%$ & 221 & $80,7 \%$ & \\
\hline Sistema cardiovascular & 0 & $0,0 \%$ & 274 & $100,0 \%$ & \\
\hline Sistema respiratório & 37 & $13,5 \%$ & 237 & $86,5 \%$ & \\
\hline Sistema geniturinário & 13 & $4,7 \%$ & 261 & $95,3 \%$ & \\
\hline Sistema nervoso & 13 & $4,7 \%$ & 261 & $95,3 \%$ & \\
\hline Sistema tegumentar & 4 & $1,5 \%$ & 270 & $98,5 \%$ & $<0,0001^{*}$ \\
\hline Sistema linfático & 11 & $4,0 \%$ & 263 & $96,0 \%$ & \\
\hline Sistema endócrino & 0 & $0,0 \%$ & 274 & $100,0 \%$ & \\
\hline Sistema ósseo & 83 & $30,3 \%$ & 191 & $69,7 \%$ & \\
\hline Ausente & 2 & $0,7 \%$ & 272 & $99,3 \%$ & \\
\hline Não informado & 37 & $13,5 \%$ & 237 & $86,5 \%$ & \\
\hline \multicolumn{6}{|l|}{ Tratamento realizado } \\
\hline Não & 25 & $9,1 \%$ & 249 & $90,9 \%$ & \\
\hline Cirurgia & 62 & $22,6 \%$ & 212 & $77,4 \%$ & \\
\hline Quimioterapia & 106 & $38,7 \%$ & 168 & $61,3 \%$ & \\
\hline Radioterapia & 126 & $46,0 \%$ & 148 & $54,0 \%$ & $<0,0001^{*}$ \\
\hline Braquiterapia & 9 & $3,3 \%$ & 265 & $96,7 \%$ & \\
\hline Hormonioterapia & 3 & $1,1 \%$ & 271 & $98,9 \%$ & \\
\hline Não informado & 77 & $28,1 \%$ & 197 & $71,9 \%$ & \\
\hline \multicolumn{6}{|l|}{ Equipe multidisciplinar } \\
\hline Assistente social & 263 & $96,0 \%$ & 11 & $4,0 \%$ & \\
\hline Psicólogo & 251 & $91,6 \%$ & 23 & $8,4 \%$ & \\
\hline Enfermeiro & 254 & $92,7 \%$ & 20 & $7,3 \%$ & \\
\hline Fisioterapeuta & 26 & $9,5 \%$ & 248 & $90,5 \%$ & \\
\hline Nutricionista & 2 & $0,7 \%$ & 272 & $99,3 \%$ & 0,43 \\
\hline Médico & 260 & $94,9 \%$ & 14 & $5,1 \%$ & \\
\hline Fonoaudiólogo & 12 & $4,4 \%$ & 262 & $95,6 \%$ & \\
\hline Terapeuta ocupacional & 43 & $15,7 \%$ & 231 & $84,3 \%$ & \\
\hline Odontólogo & 2 & $0,7 \%$ & 272 & $99,3 \%$ & \\
\hline
\end{tabular}

Legendas: Teste do qui-quadrado (contingência). ${ }^{*}$ Resultado estatisticamente significante, $\mathrm{p} \leq 0,05$. 
Tabela 5. Distribuição do uso de medicamentos pelos pacientes com câncer, atendidos pelo programa de visita domiciliar do Hospital Ophi Loyola, Belém, Pará, 2010 a 2017 ( $\mathrm{n}=274$ )

\begin{tabular}{|c|c|c|c|c|}
\hline \multirow{2}{*}{ MEDICAMENTOS } & \multicolumn{2}{|c|}{ Sim } & \multicolumn{2}{|c|}{ Não } \\
\hline & $\mathbf{N}$ & $\%$ & $\mathbf{N}$ & $\%$ \\
\hline $\begin{array}{l}\text { Analgésico de alta } \\
\text { potência }\end{array}$ & 148 & $54,0 \%$ & 126 & $46,0 \%$ \\
\hline $\begin{array}{l}\text { Analgésico de } \\
\text { baixa potência }\end{array}$ & 42 & $15,3 \%$ & 232 & $84,7 \%$ \\
\hline $\begin{array}{l}\text { Antiácido e } \\
\text { antiulcerativo }\end{array}$ & 93 & $33,9 \%$ & 181 & $66,1 \%$ \\
\hline Antiemético & 93 & $33,9 \%$ & 181 & $66,1 \%$ \\
\hline Antipsicótico & 82 & $29,9 \%$ & 192 & $70,1 \%$ \\
\hline Diurético & 8 & $2,9 \%$ & 266 & $97,1 \%$ \\
\hline Antidepressivo & 53 & $19,3 \%$ & 221 & $80,7 \%$ \\
\hline Antimicrobiano & 17 & $6,2 \%$ & 257 & $93,8 \%$ \\
\hline Corticosteroide & 25 & $9,1 \%$ & 249 & $90,9 \%$ \\
\hline Anticonvulsivante & 15 & $5,4 \%$ & 256 & $93,4 \%$ \\
\hline Laxante & 78 & $28,4 \%$ & 196 & $71,5 \%$ \\
\hline Anti-hipertensivo & 17 & $6,2 \%$ & 257 & $93,8 \%$ \\
\hline Benzodiazepínico & 26 & $9,4 \%$ & 248 & $90,5 \%$ \\
\hline $\begin{array}{l}\text { Analgésico e } \\
\text { antitérmico }\end{array}$ & 34 & $12,4 \%$ & 240 & $87,6 \%$ \\
\hline Antifibrinolítico & 3 & $1,0 \%$ & 271 & $98,9 \%$ \\
\hline Antidiabético & 2 & $0,7 \%$ & 272 & $99,3 \%$ \\
\hline AINE & 6 & $2,1 \%$ & 268 & $97,8 \%$ \\
\hline Anticonvulsivante & 3 & $1,0 \%$ & 271 & $98,9 \%$ \\
\hline Antifisético & 4 & $1,5 \%$ & 270 & $98,5 \%$ \\
\hline Anti-hemorrágico & 8 & $2,9 \%$ & 266 & $97,1 \%$ \\
\hline Anti-histamínico & 1 & $0,3 \%$ & 273 & $99,6 \%$ \\
\hline Anticolinérgico & 1 & $0,3 \%$ & 273 & $99,6 \%$ \\
\hline Expectorante & 2 & $0,7 \%$ & 272 & $99,3 \%$ \\
\hline Antiflatulento & 2 & $0,7 \%$ & 272 & $99,3 \%$ \\
\hline Antiarrítmico & 1 & $0,3 \%$ & 273 & $99,6 \%$ \\
\hline
\end{tabular}

dos cuidados para doenças neoplásicas, que, atualmente, representam a segunda causa de óbito no país ${ }^{25}$, além da necessidade de investimento no nível primário para prevenção das neoplasias, diagnóstico precoce e tratamento adequado imediato.

Para os casos de câncer metastático, a metástase óssea é uma ocorrência frequente, sendo o terceiro local mais comum de metástases distantes, seguido do fígado e pulmão. Existem casos em que as metástases ósseas são indolores, porém, em muitos casos, ocasionam dor significativa e debilitante ${ }^{26}$. Além da dor óssea, as metástases ósseas podem causar outras comorbidades, como fraturas patológicas e compressão da medula espinha ${ }^{26}$, dado similar ao deste trabalho, cujo local de maior incidência de metástase foi no sistema ósseo.

Nas neoplasias de pulmão, mama, próstata e no mieloma, a invasão tumoral óssea é a causa mais comum, sendo um tipo de dor nociceptiva somática em que o mecanismo acorre quando o tumor ativa os nociceptores por pressão, levando à isquemia e à secreção de substâncias álgicas como prostaglandinas e fator de ativação de osteoclastos $^{27}$.

A dor foi o principal sintoma na populaçáo estudada, corroborando a literatura produzida por Rangel e Telles ${ }^{27}$, na qual foi identificado que a dor acomete de $60 \%$ a $80 \%$ dos pacientes com câncer; destes, $25 \%$ a $30 \%$ no momento do diagnóstico e $70 \%$ a $90 \%$ dos pacientes com estadiamento avançado que classificaram a dor como moderada a grave.

Quanto à abordagem medicamentosa para o alívio da dor, a escolha do opioide mais adequado deve estar, primeiramente, de acordo com a intensidade da dor. Nessa perspectiva, pesquisa realizada por Fripp ${ }^{25}$, sobre o perfil clínico de 213 pacientes do Programa de Internação Domiciliar Interdisciplinar, verificou que $65 \%$ dos pacientes referiram dor; destes, $90 \%$ relataram dor moderada a intensa. Do total de pacientes, $78 \%$ necessitaram de analgésicos opioides, sendo os mais utilizados morfina (43\%) e codeína (26\%). Ainda sobre os opioides disponíveis, destaca-se a morfina, considerada padrão-ouro no tratamento da dor moderada e intensa, sendo indicada em outros estudos na literatura ${ }^{28,29}$.

\section{CONCLUSÃO}

Este estudo analisou a caracterização clínica e sociodemográfica dos pacientes em cuidados paliativos em assistência domiciliar. Os dados encontrados neste trabalho foram similares aos encontrados na literatura e mostraram predominância do sexo feminino, com idade média variando de 60 a 80 anos, baixo nível socioeconômico e tempo de estudo de quatro a sete anos, a maioria era Paraense e pertencente à cidade de Belém.

Quanto ao perfil clínico, o câncer de maior frequência se localizava no sistema geniturinário, com estadiamento clínico avançado e grandes ocorrências de metástases, principalmente para o sistema ósseo. A dor foi um sintoma encontrado na maioria da população e, entre as medicaçóes mais utilizadas, a classe terapêutica de analgésicos de ampla potência foi a mais comum.

Diante disso, finaliza-se este trabalho salientando a importância de se conhecer o perfil clínico e sociodemográfico dos pacientes, para melhor planejar e intervir nesse público junto à equipe multidisciplinar, por meio de materiais consultivos (planilhas) e pesquisas 
epidemiológicas, visto que a população deste estudo tem um cenário de alta prevalência de tipos de câncer que podem ser diagnosticados precocemente. Faz-se necessário o incentivo direto a esse público com medidas de açôes preventivas, de promoção, diagnóstico precoce e tratamento, proporcionadas pelo poder público local, o que consequentemente poderá contribuir para a regressão de casos paliativos.

Como limitaçôes deste trabalho, pode-se citar o fato de ainda haver uma escassez de estudos e pesquisas científicas acerca do tema em questão nessa Regiâo, assim como daqueles que abordem o perfil clínico e sociodemográfico de pacientes que estejam em cuidados paliativos em assistência domiciliar.

\section{CONTRIBUIÇÕES}

Todas as autoras contribuíram na concepção el ou no planejamento do estudo; na obtençáo, análise e interpretação dos dados; assim como na redação e revisão crítica; e aprovaram a versão final a ser publicada.

\section{DECLARAÇÃO DE CONFLITO DE INTERESSES}

Nada a declarar.

\section{FONTES DE FINANCIAMENTO}

Não há.

\section{REFERÊNCIAS}

1. Santos M, Matsudo VKR. Atividade física e uso de medicamentos. Diagn Tratamento. 2018;23(4):152-159.

2. Macêdo GD, Lucena NMG, Soares LMMM, et al. Influência do estilo de vida na qualidade de vida de mulheres com câncer de mama. Rev Bras Cienc Saude. 2011;14(4):13-18.

3. Batista DRR, Mattos M, Silva SF. Convivendo com o câncer: do diagnóstico ao tratamento. Rev Enferm UFSM 2015;5(3):499-510. doi: http://doi. org/10.5902/2179769215709

4. Carvalho RT, Parsons HA, organizadores. Manual de cuidados paliativos ANCP. 2. ed. São Paulo: Academia Nacional de Cuidados Paliativos; 2012.

5. Menezes MFB, Camargo TC, Guedes MTS, et al. Câncer, pobreza e desenvolvimento humano: desafios para a assistência de enfermagem em oncologia. Rev Latino Am Enfermagem. 2007;15(n. spe):780-85. doi: https://doi.org/10.1590/S0104-11692007000700011

6. Paiva CJK. Câncer de mama no hospital de câncer de Pernambuco: perfil sociodemográfico e aspectos relacionados ao atraso no diagnóstico e tratamento [dissertação]. Recife: Departamento de Saúde Coletiva, Instituto Aggeu Magalhães, Fundação Oswaldo Cruz; 2013.

7. Kolankiewicz ACB, Souza MM, Magnago TSBS, et al. Apoio social percebido por pacientes oncológicos e sua relação com as características sociodemográficas. Rev Gaúcha Enferm. 2014;35(1):31-38. doi: http://doi. org/10.1590/1983-1447.2014.01.42491

8. Saunders CM. Cicely Saunders: selected writings 19582004. Oxford: Oxford University Press; 2006. Chapter 42, The evolution of palliative care; p. 263-8.

9. Floriani CA, Schramm FR. Desafios morais e operacionais da inclusão dos cuidados paliativos na rede de atenção básica. Cad Saúde Pública. 2007;23(9):2072-80. doi: https://doi.org/10.1590/S0102-311X2007000900015

10. Santos LR, Ponce de Leon CGRM, Funghetto SS. Princípios éticos como norteadores no cuidado domiciliar. Ciênc Saúde Colet. 2011;16(Suppl 1):855-63. doi: https://doi.org/10.1590/S1413-81232011000700017

11. Ministério da Saúde (BR). Portaria no 825, de 25 de abril de 2016. Redefine a Atençáo Domiciliar no âmbito do Sistema Único de Saúde (SUS) e atualiza as equipes habilitadas. Diário Oficial da Uniāo. Brasília, DF; 2016 abr 26. Secção 1, p.33.

12. Vasconcellos MM, Gribel EB, Moraes IHS. Registros em saúde: avaliação da qualidade do prontuário do paciente na atenção básica. Cad Saúde Pública. 2008;4(Suppl 1):s173-s182. doi: http://doi.org/10.1590/S0102311X2008001300021

13. Conselho Nacional de Saúde (BR). Resolução no 466, de 12 de dezembro de 2012. Aprova as diretrizes e normas regulamentadoras de pesquisas envolvendo seres humanos. Diário Oficial da União, Brasília, DF; 2013 jun. 13. Seção I, p. 59.

14. Bastos BR, Pereira AKS, Castro CC, et al. Perfil sociodemográfico dos pacientes em cuidados paliativos em um hospital de referência em oncologia do estado do Pará, Brasil. Rev Pan-Amaz Saúde. 2018;9(2):31-6. doi: http://doi.org/10.5123/s2176-62232018000200004

15. Souza RS, Simão DAS, Lima EDRP. Perfil sociodemográfico e clínico de pacientes atendidos em um serviço ambulatorial de quimioterapia paliativa em Belo Horizonte. Rev Min Enferm. 2012;16(1):38-47.

16. Carnaúba CMD, Silva TDA, Viana JF, et al. Caracterização clínica e epidemiológica dos pacientes em atendimento domiciliar na cidade de Maceió, AL, Brasil. Rev Bras Geriatr Gerontol 2017;20(3):353-63. doi: https://doi. org/10.1590/1981-22562017020.160163

17. Ciałkowska-Rysz A, Kowalczyk M, Gottwald L, et al. The comparison of common cancer types and the coincidence of concomitant chronic diseases between palliative home care patients in Lodz Voivodeship and the general Polish population. Arch Med Sci. 2012;8(3):496-503. doi: https://doi.org/10.5114/aoms.2012.29406 
18. Silva JVF, Silva EC, Rodrigues APRA, et al. A relação entre o envelhecimento populacional e as doenças crônicas não transmissíveis: sério desafio de saúde pública. Ciên Biol Saúde. 2015;2(3):91-100.

19. Lampert MA, Brondani CM, Donati L, et al. Perfil de doentes crônicos de um serviço de internação domiciliar da Região Sul do Brasil. J Nurs Health. 2013;3(2):14756. doi: https://doi.org/10.15210/JONAH.V3I2.3554

20. Malta DC, Andrade SSCA, Oliveira TP, et al. Probabilidade de morte prematura por doenças crônicas não transmissíveis, Brasil e Regiōes, projeçôes para 2025. Rev Bras Epidemiol. 2019;22: e190030. doi: https://doi. org/10.1590/1980-549720190030

21. Instituto Nacional de Câncer José Alencar Gomes da Silva. Estimativa 2020: incidência de câncer no Brasil [Internet]. Rio de Janeiro: INCA; 2019 [acesso 2019 dez 11]. Disponível em: https://www.inca.gov.br/sites/ ufu.sti.inca.local/files/media/document/estimativa-2020incidencia-de-cancer-no-brasil.pdf

22. Atty ATM, Tomazelli JG. Cuidados paliativos na atenção domiciliar para pacientes oncológicos no Brasil. Saúde Debate. 2018;42(116):225-36. doi: http://doi. org/10.1590/0103-1104201811618

23. Carvalho MWA, Araújo AA, Nóbrega MML. Diagnóstico de enfermagem para pacientes com toxicidade hematológica pós-quimioterapia antineoplásica com base na CIPE. Rev Enferm UFPE. 2009;3(4):1219. doi: http://doi.org/10.5205/reuol.581-3802-1RV.0304200902

24. Visentin A. Avaliação da qualidade de vida e sobrevida de pacientes com câncer avançado na terapêutica paliativa [tese]. Curitiba: Universidade Federal do Paraná; 2016.

25. Fripp JC. Internação domiciliar e cuidados paliativos para pacientes oncológicos no município de Pelotas/RS [dissertação]. Rio Grande do Sul: Universidade Federal de Pelotas; 2009.

26. Sze WM, Shelley M, Held I, et al. Palliation of metastatic bone pain: single fraction versus multifraction radiotherapy. Cochrane Database Syst Rev. 2002(2):CD004721. doi: http://doi.org/10.1002/14651858.CD004721

27. Rangel O, Telles C. Tratamento da dor oncológica em cuidados paliativos. Rev Hosp Univ Pedro Ernesto. 2012;11(2):32-7.

28. Fripp JC, Facchini LA, Silva SM. Caracterização de um programa de internação domiciliar e cuidados paliativos no município de Pelotas, Estado do Rio Grande do Sul, Brasil: uma contribuição à atenção integral aos usuários com câncer no Sistema Único de Saúde, SUS. Epidemiol Serv Saúde. 2012;21(1):1679-4974. doi: http://doi. org/10.5123/S1679-49742012000100007
29. Nogueira FL, Sakata RK. Palliative sedation of terminally ill patients. Rev Bras Anestesiol. 2012;62(4):580-92. doi: http://doi.org/10.1016/S0034-7094(12)70157-5

Recebido em 1/6/2020 Aprovado em 10/11/2020 